\title{
Subject and Object Index
}

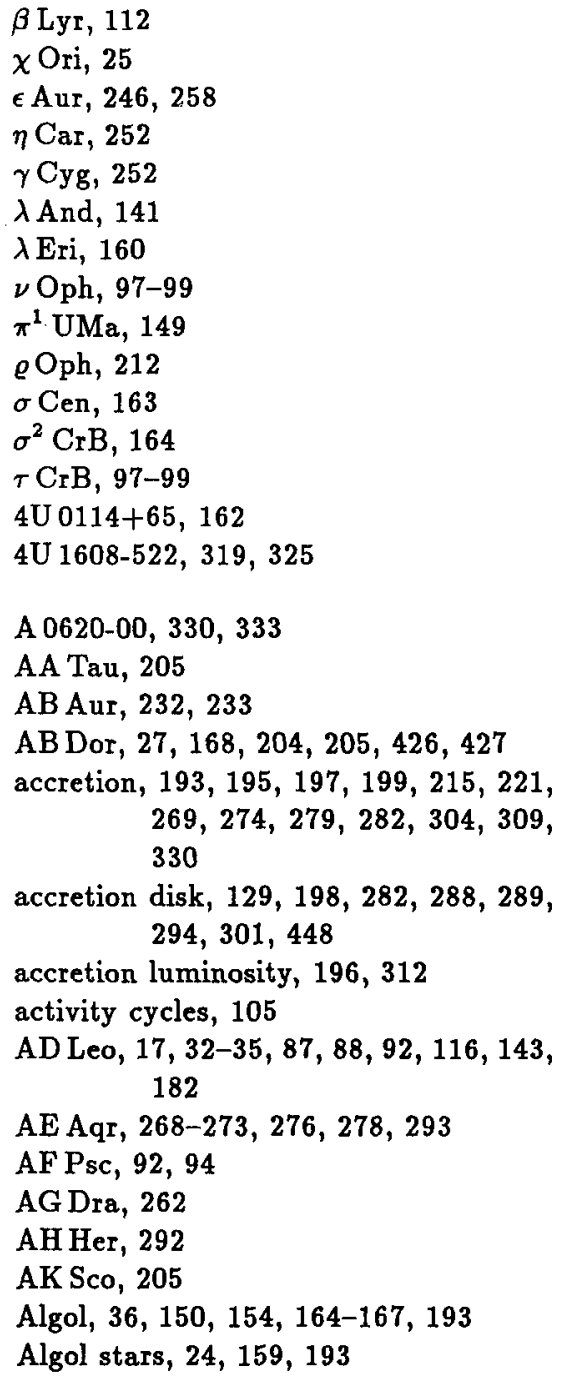

Alpha Persei cluster, 122

AM Her, 92, 302

Aql X-1, 320

AR Lac, 150, 164

ASCA satellite, 17, 156, 168, 318, 431

ASTRON satellite, 80

Atoll sources, 321

AU Mic, 15, 143, 146, 160

AXAF satellite, 156

$\mathrm{B}[\mathrm{e}]$ stars, 227

B 13, 219

B 31, 417

B 32, 417

B 32A, 417

B 41, 219

BACODINE, 341, 348-352, 370, 372, $379,385-387,394,442$

BATSE, 342-345, 349, 369, 370, 381, $385,441-444$

$\mathrm{BD}+47^{\circ} 819,101,102$

$\mathrm{BD}+65^{\circ} 1637,223$

black hole, 131, 321, 322, 324, 326, 334

BM And, 205

boundary layer, 294

BP Tau, 221

bremsstrahlung, 179, 180, 196, 197, 296

BV Cen, 292

BW Aqr, 162

BY Cam, 303

BY Dra, 15, 103, 105, 139

BY Dra stars, 24

Castor, 157

cataclysmic variables, $195,237,264,278$, $280,288,296,302,448$ 
CC Eri, 150, 170

CF Tuc, 151, 164, 429

CH Cyg, 260

Chamaeleon, 212

Chandrasekhar limit, 239

chaotic variability, $82,218,342$

CI Tau, 205, 219

Cir X-1, 325

CN Com, 106, 107

CO Ori, 205

Coma, 122

COMPTEL, 347, 351, 369

Compton GRO, 197, 342, 358, 369, 385

Compton scattering, 197-200

coronal mass ejection, 3, 146, 193, 195

Crab pulsar, 131

CT Com, 70

CU Cnc, 122

CV Cha, 205

CV Com, 70

CYMus, 162

cyclotron radiation, 197, 296

Cyg X-1, 323, 325

CZ Cnc, 94

dark-spot model, 103

DD Ser, 232, 233

DF Tau, 205, 219

DG Tau, 205, 219

DH Tau, 205

DI Cep, 205

DI Tau, 205

diffusion, 5, 200, 240

DK Tau, 205

DL Tau, 205

DM UMa, 163

DN Tau, 205

DO Tau, 205

DoAr 21, 205

DoAr 51, 205

DR Tau, 205

dwarf novae, $159,264,282,285,286$, 292

eclipse mapping, 294, 300

EGRET, 197, 342, 345, 347, 351, 352, $358,360,361,369,371,374$, 441,443

Einstein satellite, 17, 164, 170, 171, 203, $212,216,320$
EK Dra, 24-26, 29, 31

electron cyclotron maser, 32,89

emission measures, 137

EQ $1839.6+8002,171-173$

EQPeg, 28

ETA satellite, 370

ETC camera, 351, 372, 385-387, 444

EUVE satellite, $73,146,160,169,371$, 419

EV Lac, 73-80, 82-85, 92, 110, 111, 133, $134,150,155,160$

EX Hya, 292

EX Lup, 205

EXO 040830-7134.7, 159

EXOSAT satellite, 17, 149, 159, 164, 170,171

FF Tau, 219

FK Com stars, 14

flare mass motions, $137,178,183$

flares, 3, 14-17, 19, 23, 36, 47, 73, 87, $89,91,97,101,110,119,121$, $164,177,212,216,279,286$, 423,448

flare transition regions, 137

flare TR electron densities, 137

flickering, 260, 278, 279, 288, 300, 447, 448

FU Ori, 245

FY Aql, 371

FY Tau, 218, 219

FZ Tau, 218, 219

G102-21, 92, 95, 96

gamma-ray bursts, $91,93,341-346,353$, $358,363,367,376,381,385$, $389,393,400,431,441$

GB 781006B, 91, 93

GB 790107, 368

GB 790305b, 368

GB 790324, 368

GB 790331, 371

GB 790418, 389-391

GB 790613, 389-391

GB 830313,377

GB 900222, 355

GB 900320, 355

GB 910503, 361

GB 910601, 361

GB 920325, 371 
GB 920501, 372

GB 920723, 354, 355

GB 930131, 361, 372

GB 940217, 361, 371

GB 940301, 361, 372, 373

GB 941014, 385, 387, 388

GBS $0008+13,91,93$

GBS 0855-00, 368

Geminga, 419

GG Tau, 205

GITau, 205, 219

Ginga satellite, 150, 159, 164, 171, 204, 323,431

GK Tau, 205

Gl $166 \mathrm{C}, 59$

Gl 412B, 59

Gl 424,59

Gl $630.1,59$

Gl 781, 59

Gl 866, 59

Gl 867B, 116

GN Tau, 219

GQ Lup, 205

GQ Mus, 239

Granat satellite, 353, 356

GRO J0422+32, 132, 330, 332, 333

GROCSE, 351, 370, 372

Groombridge 1830B, 59

GRS 0818-52, 160

GRS 1100-77, 158, 160, 161

GRS $1133+54,160$

GRS $1148-66,160,162$

GRS $1624-37,160,162$

GRS 2037-40, 160, 162

GRS 2220-15, 160, 162

GS 1124-68, 323

GS $2023+338,326$

GV Tau, 217

GW Lup, 205

GW Ori, 205

GX $17+2,322$

GX 339-4, 326, 334

gyrosynchrotron emission, 23, 36, 40

Haro 1-16, 205

HD 129333, 24

HD 147365,27

HD 152678, 108

HD 16157, 170

HD 197890, 428
HD 283447, 205

HD $36705,426,427$

HD 5303, 429

HD 560B, 204

HDE 259431, 227

He-burning, 319

helium shell flash, 237, 240

Her X-1, 419

Herbig Ae/Be stars, 227

HETE satellite, 370, 394, 444

high speed photometry, 131, 221, 330

HII 1136, 27

HK Aqr, 139

HO Com, 70

HP Tau, 205, 219

HP Tau G2, 205

HQ Com, 70

HQ Tau, 219

HR $1099,28,36,37,39-41,44,159,426$, 427

HR 5110, 36

HR 963, 31

HR Del, 292

HS Com, 70

HST, 137

HT Cas, 292, 294, 300, 301

HV Com, 70

HV Tau, 219

Hyades, 212

$\mathrm{Hz} 2411,214$

HZ Her, 400

IC 1318, 122

IC 2391, 212

IC 348, 212

II Peg, 15, 17, 140, 141, 150

IM Peg, 141

IMB detector, 374

IPN, 347, 351, 369

IUE satellite, 18, 73, 137-139, 142, 143, $145,238,280,282$

IX Oph, 205

KK Oph, 205

KM Ori, 205

KN Ori, 205

KR Aur, 290, 291, 400

LH $\alpha 332-17,205$

LH 332-20, 161 
LIGO detector, 374

$\mathrm{LkH} \alpha 118,205$

$\mathrm{LkH} \alpha 234,223$

LMC X-1, 334

LMC X-3, 334

loss-cone instabilities, 23, 35

low-mass X-ray binaries, $163,195,196$, $319,321,330$

M15, 317

M42/43, 119, 122

magnetic activity, 149, 195

Markov chain, 291

mass loss rate, 195

M dwarf flare stars, $14,15,17,32,47$, $51,55,57,61,63,65,67,69$, $87,91-93,95,115,119,121$, $125,129,131,137-144,148$, $149,151,159,160,171,173$, $177,185,212,416,441,444$, $445,447-449$

MILAGRO detector, 372

monitoring: optical, 115, 402

Mont Blanc detector, 374

MR Ser, 302

MV Lyr, 303

MWC 137, 227

MWC 342, 227

MWC 349, 228

MX 2346-65, 158

MXB 1735-44, 132, 330, 332, 333

neutron stars, $129,243,274,309-312$, $316,317,319,321,324,326$, 419,448

NGC 2264, 122, 219

NGC 7000, 122, 219

NGC 7129, 223

noise, 290, 321-324, 326

novae, $237,264,447$

OPAL opacity, 237

optical transients, 91, 93, 367, 376, 381, $385,389,393,400,441,443$, 444

Orion, 212, 219

OY Car, 286

plate collection, $397,402,407,412,416$, 441
Pleiades, 122, 212

PMS stars, 149

power spectra, 277, 290, 322, 323, 325

Praesepe, 122

prefiltering technique, 133

Procyon, 26

Proxima Cen, 62, 156, 164

PSR 0656+14, 419

PSR $1937+21,131$

PSR J0437-4715, 419, 421

PU Vul, 245-253, 255, 257

quasi-periodic oscillations, $277,303,319$, 321

R CrB, 249

R Mon, 205

radio continuum emission, 23

radio flares, $23,32,40,42,44,89,177$, 268

Rapid Burster, 319

RE J0241-53N, 67, 68

RE J0604-34, 68

RE J0625-60, 68

RE J2055-17B, 68

reconnection, $3-8,11,12,146,150,154-$ $156,170,177,197,198,200$

ROSAT satellite, 17, 24, 29, 67, 69, 79, $87,89,149,160,164,165,167$, $170,171,212,216,423-429$

ROX 20, 161

ROX 3, 205

RR Pic, 239

RR Tel, 252

RS CVn stars, 14, 15, 18, 19, 24, 36, 40, $106,107,137,140,141,144$, $145,148-150,159,162$

RV Mic, 162

RW Aur, 203, 205

RW Tri, 300

RX And, 292

RX J1219.7+1630, 106

RX J1235.5+1954, 69-71

RX J1253.6+2247, 69-71

RX J1256.8+2329, 69-71

RX J1258.9+2112, 69-71

RX J1300.5+2255, 70, 71

RY Sct, 112

RY Tau, 205, 207, 219 
S 10933, 91, 92, 94
S 10935, 69-71
S 10936, 69-71
S 10937, 69-71
S 10938, 69-71
S 10939, 70, 71
S CrA, 205
Sco X-1, 289, 322

Scorpius-Ophiuchus, 122

shear mixing, 240

shot noise, 296, 304

SMM satellite, 4, 180, 371

soft gamma-ray repeaters, $346,368,431$

solar flare frequency, 3,45

solar X-ray emission, 3, 148

Sonneberg Observatory, 69, 91, 106, 107, 203, 207, 229, 262, 381, 399, $400,402,412,451-454$

Soudan detector, 374

SR 12, 205

SR 4, 205

SR 9, 205

SS Aqr, 162

SS Cyg, 283, 292

star clusters, $47,55,63,65,122,212$, 216

SU Aur, 205

SU Tau, 219

SU UMa stars, 265

Sun, $3,14,15,18,35,40,45,47,77$, $139,148,149,151,152,155$, $170,177,195,212,353$

sunspots, $9,13,45$

supernovae type Ia, 241

supernova type Ia, 448

SVS 1099, 219

SY Cnc, 292

SY Tau, 205

symbiotic novae, 245

synchrotron radiation, $129,197,269$

Sz 68, 205

SZ Cha, 205

T Cha, 205

T CrB, 239

T Tau, 205, 218, 219

T Tau stars, 14, 24, 47, 51, 55, 63, 158, $203,212,216,218,221,289$

Taurus dark cloud, 122

TiO band, 86, 111
TT Ari, 288, 289, 302, 303

T'T Lupi, 157

TUMen, 286, 287

TW Cha, 205

TW Hya, 205

two-ribbon flares, $3,150,164,170$

TY Mus, 162

U Cep, 193

$\mathrm{U}$ CrB, 193

U Gem, 300

U Gem stars, 265

u Her, 193

U Mic, 162

U Sco, 239

UV Cet, 89, 92, 116

UV Mus, 162

UV sources, 67, 241, 243, 282, 419, 444

UV spectroscopy, 137, 282

UX Ari, 36, 42, 150, 159

UX Tau A, 205

UX UMa stars, 288

V1005 Ori, 17, 139

V1036 Tau, 417

V1054 Oph, 108, 109

V1118 Ori, 229-231

V1143 Ori, 229-231

V1194 Ori, 417

V1263 Ori, 418

V1297 Ori, 418

V1500 Cyg, 253

V1668 Cyg, 238

V2051 Oph, 292

V350 Cep, 223

V367 Cyg, 112

V370 Ori, 205

V371 Ori, 76, 77

V394 CrA, 239

V410 Tau, 219

$\mathrm{V} 426 \mathrm{Oph}, 292$

V436 Cen, 292

V442 Cen, 286

V518 Per, 132

V590 Tau, 219

V603 Aql, 292

V616 Mon, 132

V654 Her, 97-99

V711 Tau, 17, 28, 37, 39, 44, 141, 426, 427 


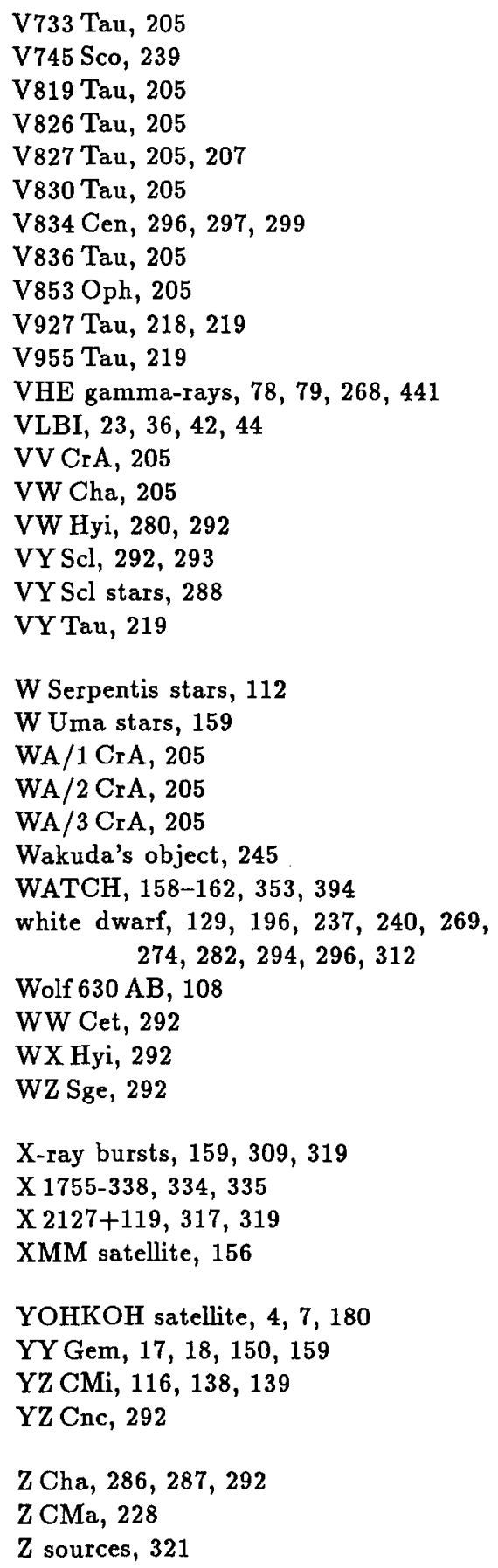

